\title{
27 years of prenatal diagnosis for Huntington disease in the United Kingdom
}

\author{
Raul E. Piña-Aguilar, $\mathrm{MD}^{1,2}$, Sheila A. Simpson, MBChB, MD², Abdulrahman Alshatti, MSc ${ }^{1,18}$, \\ Angus Clarke, BM BCh, DM" ${ }^{3}$, David Craufurd, MBBS, MSc, ${ }^{4,5}$, Huw Dorkins, BMChB, $\mathrm{MSc}^{6}$, \\ Karen Doye, RN, BSc ${ }^{7}$, Nayana Lahiri, MBBS, MD ${ }^{8,9}$, Alison Lashwood, RN ${ }^{10}$, Colleen Lynch, BSc, MSc ${ }^{11}$, \\ Claire Miller, $\mathrm{RN}^{12}$, Sally Morton, BSc, MSc ${ }^{13}$, Mary $\mathrm{O}^{\prime}$ Driscoll, MBBS ${ }^{14}$, \\ Oliver W. Quarrell, MBBS, MD ${ }^{15}$, Daniela Rae, RN, MSc ${ }^{1,2}$, Mark Strong, PhD ${ }^{16}$, \\ Charlotte Tomlinson, BSc, MSc ${ }^{10}$, Peter Turnpenny, MBBS ${ }^{17}$ and \\ Zosia Miedzybrodzka, MBChB, $\mathrm{PhD}^{1,2}$ on behalf of the UK HD Predictive Testing Consortium
}

\begin{abstract}
Purpose: There is little long-term, population-based data on uptake of prenatal diagnosis for Huntington disease (HD), a lateonset autosomal dominant neurodegenerative disorder, and the effect of the availability of preimplantation genetic diagnosis (PGD) on families' decisions about conventional prenatal diagnosis is not known. We report trends in prenatal diagnosis and preimplantation diagnosis for $\mathrm{HD}$ in the United Kingdom since services commenced.
\end{abstract}

Methods: Long-term UK-wide prospective case record-based service evaluation in 23 UK Regional Genetic Centres 1988-2015, and four UK PGD centers 2002-2015.

Results: From 1988 to 2015, 479 prenatal diagnoses were performed in the UK for HD. An exclusion approach was used in $150(31 \%)$. The annual rate of HD prenatal diagnosis has remained around 18 (3.5/million) over 27 years, despite a steady increase in the use of PGD for HD since 2002.

Conclusion: Although increasing number of couples are choosing either direct or exclusion PGD to prevent HD in their offspring, both direct and exclusion prenatal diagnosis remain important options in a health system where both PGD and prenatal diagnosis are state funded. At-risk couples should be informed of all options available to them, preferably prepregnancy.

Genetics in Medicine (2019) 21:1639-1643; https://doi.org/10.1038/s41436018-0367-z

Keywords: Huntington disease; chorionic villi; amniocentesis; neurodegenerative disorders; preimplantation genetic diagnosis

\section{INTRODUCTION}

Huntington disease (HD) is an autosomal dominant neurodegenerative disease characterized by cognitive decline, movement disorder, and frequent psychopathology, leading to death over 10-25 years. Onset is most often in a person between the ages of 30 and 50 years, but may be earlier or later. ${ }^{1}$ Growing up with an affected parent can present a significant psychological burden for those at risk. ${ }^{2}$ Those at risk of HD have a number of reproductive options. ${ }^{3}$ Many choose to accept the $50 \%$ risk of each child being affected; some choose to remain childless, adopt a child, or use gamete donors. The identification by linkage of the HD locus resulted in the additional option of prenatal diagnosis (PND) and termination of affected pregnancies, or increasingly, the possibility of preimplantation genetic diagnosis (PGD) to select pathogenic variant negative embryos conceived by in vitro fertilization (IVF).

Presymptomatic predictive testing and prenatal diagnosis (PND) in HD serve as models for testing in other late-onset genetic neurodegenerative disorders, such as familial early-

\footnotetext{
${ }^{1}$ School of Medicine, Medical Sciences and Nutrition, University of Aberdeen, Aberdeen, UK; ${ }^{2}$ North of Scotland Regional Genetics Service, Aberdeen Royal Infirmary, Aberdeen, UK; ${ }^{3}$ Institute of Cancer and Genetics, University of Cardiff, Cardiff, UK; ${ }^{4}$ Manchester Centre for Genomic Medicine, Division of Evolution and Genomics Sciences, School of Biological Sciences, Faculty of Biology, Medicine and Health, University of Manchester, Manchester, UK; ${ }^{5}$ St. Mary's Hospital, Central Manchester University Hospitals NHS Foundation Trust, Manchester, UK; ${ }^{6}$ Leicester Royal Infirmary, University Hospitals of Leicester NHS Trust, Leicester, UK; ${ }^{7}$ The Centre for Reproductive \& Genetic Health, London, UK; ${ }^{8}$ Clinical Genetics department, St George's University of London, London, UK; ${ }^{9}$ St George's University Hospitals NHS Foundation Trust, London, UK; ${ }^{10}$ Clinical Genetics \& Centre for PGD, Guy's Hospital, London, UK; ${ }^{11}$ CARE Fertility Centre, Nottingham, UK; ${ }^{12}$ Cheshire and Merseyside Clinical Genetics Service, Liverpool Women's NHS Foundation Trust, Liverpool, UK; ${ }^{13}$ South East of Scotland Genetics Services, Molecular Medicine Centre, Western General Hospital, Edinburgh, UK; ${ }^{14}$ West Midlands Regional Genetics Service and Birmingham Health Partners, Birmingham Women's NHS Foundation Trust, Birmingham, UK; ${ }^{15}$ Department of Clinical Genetics, Sheffield Children's Hospital, Sheffield, UK; ${ }^{16}$ School of Health and Related Research (ScHARR), University of Sheffield, Sheffield, UK; ${ }^{17}$ Peninsula Clinical Genetics, Royal Devon \& Exeter Healthcare NHS, Exeter, UK;

${ }^{18}$ Present address: Kuwait Medical Genetics Center, Kuwait, Kuwait. Correspondence: Peter Turnpenny (peter.turnpenny@nhs.net) or Zosia Miedzybrodzka (zosia@abdn.ac.uk) Retired: Sheila A. Simpson.
} 
onset Alzheimer disease. Family-based linkage studies have been used to offer PND in the UK since 1988. Direct variant testing became possible in 1993 with the discovery that a triplet repeat expansion within exon 1 of the huntingtin gene causes the disease. ${ }^{4}$ Fetal DNA samples for PND can be taken using chorionic villus sampling (CVS) from 11 weeks' gestation, and from around 15 weeks by amniocentesis. However, the only therapeutic option for couples who are found to have a fetus at high risk is termination of pregnancy. As HD is typically a late-onset condition, prenatal testing is not offered for information only because it would be tantamount to predictive testing of a child for an adultonset condition. Genetic counseling carefully addresses these issues in reproductive decision making. In preimplantation genetic diagnosis (PGD), couples avoid the need for termination through embryo biopsy and genetic testing of embryos created prior to implantation using in vitro fertilization. ${ }^{5}$ PGD for HD was first reported in 1996 (ref. ${ }^{6}$ ).

Some couples who use reproductive technologies to avoid having a child affected by $\mathrm{HD}$ have undergone predictive testing and know that they carry the HD gene. In such cases, PND is typically performed using "direct" testing of the pregnancy for the HD gene pathogenic variant. Those at risk who wish to avoid passing on the gene but do not wish to find out their own HD status, can use an approach termed "exclusion", "indirect", or "nondisclosing" testing. Traditionally DNA markers linked to the HD gene were used to establish which grandparent contributed the HD gene passed to the fetus from the at-risk parent: the affected grandparent or their spouse. If the fetus had not inherited the gene from the affected grandparent, this excluded the mutated HD gene in the pregnancy or embryo. Exclusion testing, whether applied through PND or PGD, allows the at-risk parent to avoid discovery of their genetic risk. However, this means that parents who do not have the mutated HD gene may terminate an unaffected pregnancy or discard unaffected embryos that happen to share the affected grandparental haplotype, as there is a $50 \%$ chance that this haplotype harbors the mutated gene, and a $50 \%$ chance that it harbors the normal copy. In recent years, many services have replaced the use of linked markers in HD prenatal testing with nondisclosure of variant test results to avoid incorrect results, leading to the term "nondisclosing testing" being used in the United States. In the United Kingdom, exclusion testing using linked markers has continued in PGD to avoid incorrect results through allele dropout.

In the UK, access to PND is overseen by National Health Service (NHS) boards/trusts and professional networks, but access to PGD is regulated by the Human Fertilisation and Embryology Authority (HFEA). Before 1993, prenatal diagnosis for HD was performed using linkage analysis. Both direct and exclusion PND have been available in the UK since 1993, free at the point of care through NHS public funded health care. Direct PGD for HD was licensed in the UK from 2002, and exclusion PGD from 2009. NHS funding for direct and exclusion PGD testing for HD is now widely available free of charge to couples at risk across the UK who do not already have a child and fulfill other NHS funding criteria for PGD.

With better awareness of reproductive risks and options within families, and increasing optimism driven by global research efforts in HD treatment and better availability of PGD, we hypothesized that the request rate for PND might be in decline.

Data on HD PND have been systematically retrospectively collected from all 23 Regional Genetic Centres on an annual basis since testing began 27 years ago. Results from the first years, 1994-1998, have been reported. ${ }^{7}$ Here we describe the trends in PND in the UK since inception of these services, and compare these with available data on UK PGD uptake.

\section{MATERIALS AND METHODS Study population}

Members of the UK HD Predictive Testing Consortium retrospectively submitted annual anonymous data on prenatal diagnosis uptake from 1987 to 2015, using the same core data format for most of that time.

The number of PGD cycles performed each year for HD in the UK were reported by Guy's Hospital, the Centre for Reproductive and Genetic Health in London, the Western General Hospital in Edinburgh, and the CARE Centre in Nottingham.

\section{Ethics}

"UK predictive testing for Huntington's disease group annual prenatal testing audit" has been given formal approval by NHS Grampian (reference 3992) and the Caldicott guardian as a national audit, and therefore did not require additional institutional review board/ethics approval.

\section{Endpoints}

Numbers of cases and the types of test were recorded annually and presented at the annual HD consortium meetings. Additional data were gathered on age, sex of the at-risk partner, predictive testing status of the at-risk partner, timing of predictive testing with respect to the pregnancy, mode of prenatal diagnosis (direct or exclusion testing), results of prenatal diagnosis, and pregnancy outcome. Available PGD data included the number of couples undergoing treatment and the total number of cycles commenced.

\section{Statistical analysis}

Analysis of case frequency was performed in all cases. Detailed information was available for 411 PND cases. Data were analyzed using IBM SPSS version 23, and the Chisquared test of proportions.

\section{RESULTS}

From 1988 to 2015, 479 prenatal studies were performed across 23 UK centers. An indirect (exclusion) approach was used in 144 (31.2\%; Fig. 1). Testing rates were low before the identification of the gene in 1993, and 1994 saw the highest 


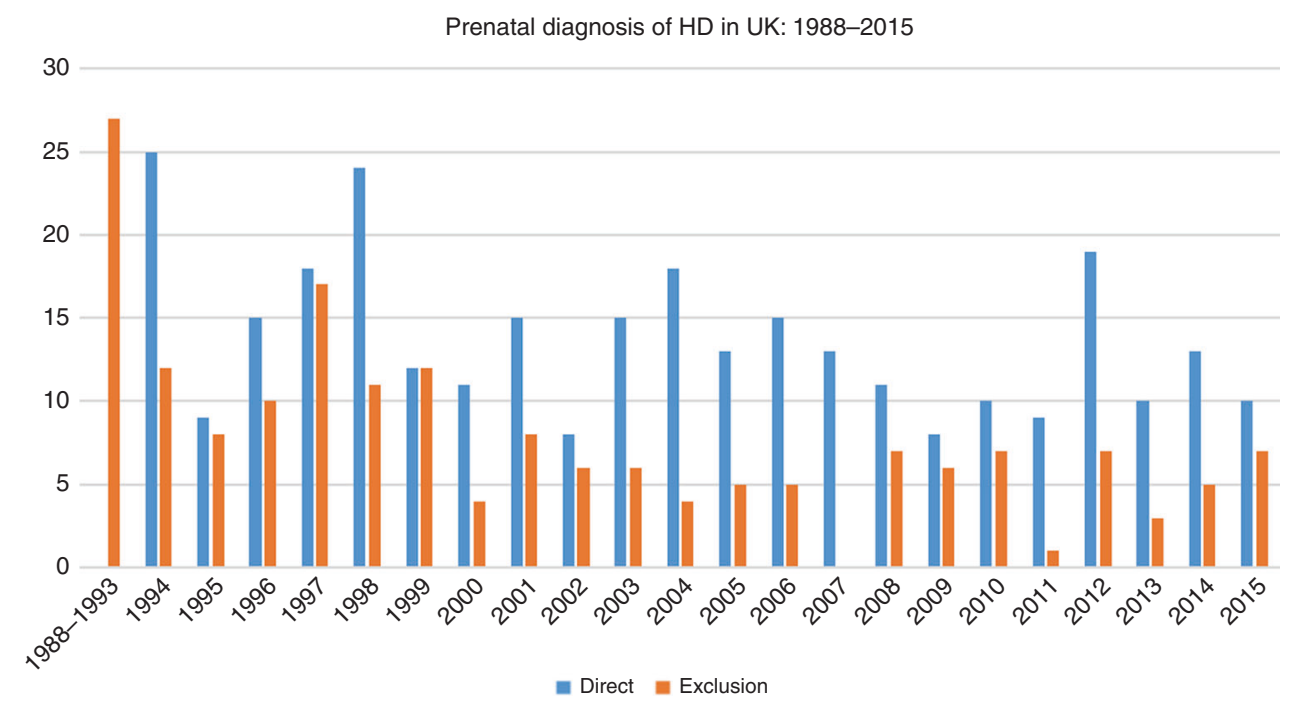

Fig. 1 Number of prenatal diagnosis cases for Huntington disease (HD) in the UK, in the period of the study (1988-2015). Orange bars refer to direct testing and blue bars to exclusion testing.

number of tests requested (37). From 1995 to 2015, the rate of PND has remained modest but steady, with a mean of 18 pregnancies per year being tested.

Detailed case information was available for 411 PND pregnancies. The at-risk parent was female in $51 \%$. Forty-five percent of the at-risk PND parents had undergone presymptomatic predictive testing and $3 \%$ had undergone diagnostic testing; only $15.8 \%$ of these predictive tests were performed during the pregnancy.

Direct variant testing was performed in $62.5 \%$ of PND, with the exclusion approach being used in $37.5 \%$. The vast majority of fetuses at $50 \%$ risk underwent a direct variant test (97\%). In contrast, where the fetal prior risk was $25 \%$ or less, an exclusion (nondisclosing) approach was used in 58\%.

The fetus was found to be affected by HD in $53 \%$ of direct PND cases, and $90.2 \%$ of these pregnancies underwent termination. The remaining $12(9.8 \%)$ were continued, resulting in the parents being aware that the child would one day develop HD. Only one HD unaffected pregnancy was reported to have been terminated. Of the exclusion tested pregnancies, termination was performed in $87.5 \%$ with a high-risk result, and the remaining 9 (12.5\%) continued. None of the low risk exclusion pregnancies underwent termination. In these 21 continued affected and high-risk pregnancies, the at-risk/affected parent was more often the mother $(56.3 \%$ vs. $43.7 \%)$, whereas in terminated pregnancies the at-risk parent was more often the father ( $52.9 \%$ vs. $47.1 \%)$, however neither of these differences reached statistical significance (two tailed Chi-square, $p=0.1616$ ).

From 2002 to 2015, 305 PGD cycles were performed in the UK for HD. The annual number of PGD cycles has increased steadily over time (Fig. 2).

\section{DISCUSSION}

PND for HD has been available in the UK since 1988. Other than a peak in the year the HD gene was identified, the rate of uptake of PND has remained modest and remarkably similar over more than two decades. The annual number of PGD cycles has steadily increased since UK licensing of the procedure in 2002.

Exclusion testing remains an important option for couples at risk, with around one-third of PND in the UK using this method. Intriguingly, available data suggest that exclusion testing is used more often in PGD (personal communication; data not shown because it is not available for all centers).

Both at-risk men and at-risk women choose PND and PGD. Around $15 \%$ of parents are symptomatic at the time of reproductive testing.

During our 27-year study period, the UK population grew from 56.93 million to 65.11 million, thus the annual rate of HD PND has fallen from to 0.316 per million to 0.276 per million. The rate of HD PGD cycles was 0.463 per million in 2015. Taking into account the prevalence and incidence of $\mathrm{HD},{ }^{8}$ we have estimated that the order of magnitude for the uptake of PND or PGD in 2015 was at least 3.0\% of at-risk pregnancies (see supplementary information). Despite the rising uptake of PGD for $\mathrm{HD}$, the vast majority of UK pregnancies at risk of HD continued to remain untested.

Access to PND and PGD funding varies worldwide. In the UK, both PND and PGD for HD is fully publicly funded for couples by the NHS and is generally coordinated through Regional Genetic Centres. Although in some areas (e.g., Scotland) PGD access is limited to couples without a child, we propose that our data indicate couples' behavior in an environment where at least some real choice is available.

As genetic diagnosis of $\mathrm{HD}$ is only provided in the UK by the NHS, our data represent a true nationwide picture, in contrast to other countries where a market in private genetic testing limits data access. A small proportion of these UK cases have been reported in a European series. ${ }^{9}$ However, only a few studies have reported national experience of PND for $\mathrm{HD}$, with reports from Canada, ${ }^{10}$ Australia, ${ }^{11}$ and the 


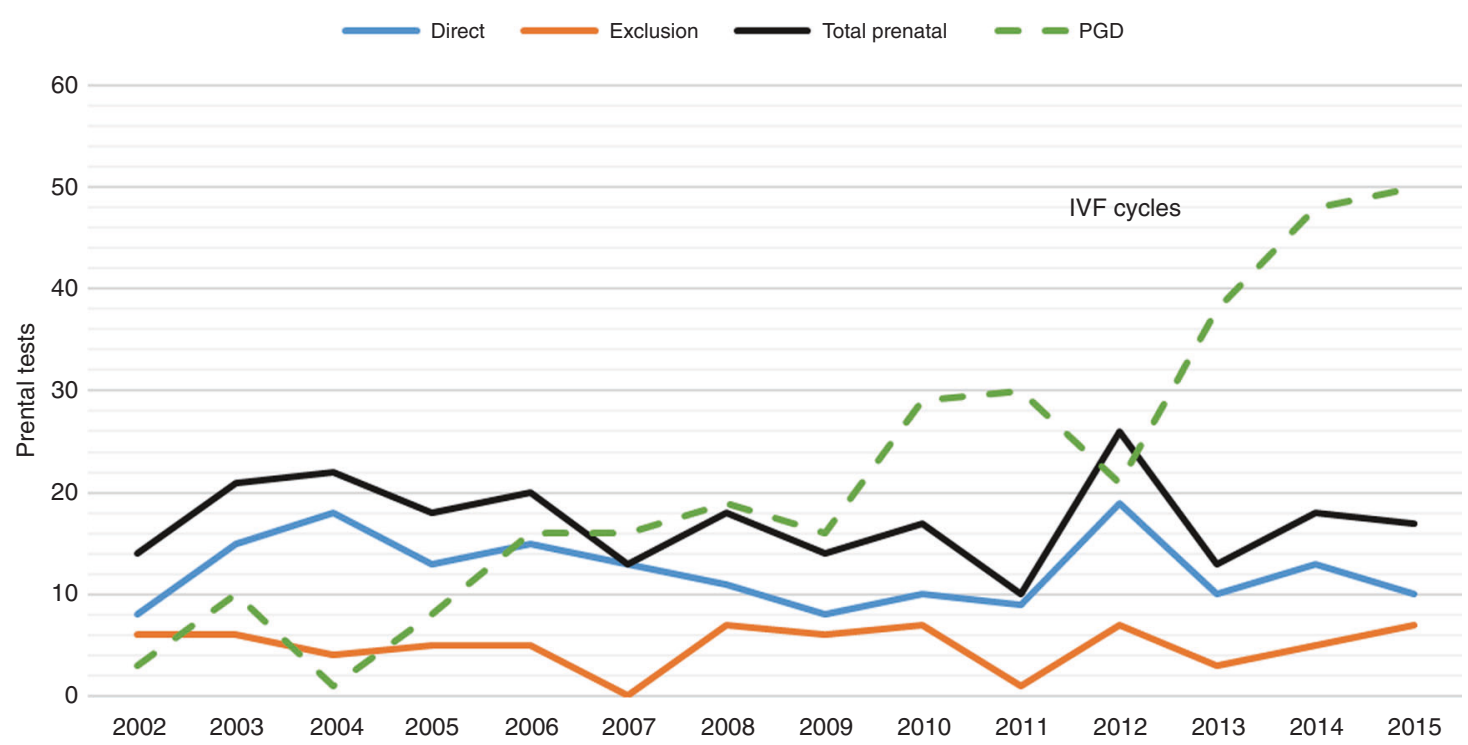

Fig. 2 Comparison of prenatal diagnosis (PND) versus preimplantation genetic diagnosis (PGD) since 2002. Orange line refers to PND direct testing, blue line exclusion testing, black line the total number of PND. Dotted green line displays the number of PGD in vitro fertilization (IVF) cycles.

Netherlands, ${ }^{12}$ but these reports did not capture the transition to PGD.

Van Rij et al. ${ }^{12}$ reported a series of 126 Dutch HD prenatal diagnoses. Eighty-two percent of affected pregnancies in that series resulted in termination, in contrast to $90 \%$ in our UK series. They estimated that in the Netherlands $22 \%$ of at-risk couples used PND when pregnant. An adjunct paper analyzing the uptake of PGD found that couples opting for PGD after pregnancy were more likely to have terminated a previous affected pregnancy than those undergoing PND alone $(87 \%$ vs. $55 \%)\left(\right.$ ref. $\left.{ }^{13}\right)$.

In the present study, other factors may play a role. In the UK, funding for both PND and PGD is provided by the NHS; although the support for PGD is more limited. NHS funding for PGD is limited to two or three cycles, or one successful pregnancy; if couples had an existing unaffected child together they would often be unable to access PGD on the NHS. There are also restrictions for NHS funding based upon the female partner's age, body mass index, and the smoking status as well as alcohol and illegal drug use of both partners. Funding thus excludes those who had successful PGD/PND previously and also those who have chosen not to have a test and therefore do not know the genetic status of their current child.

In addition, some feel that the option of PGD in the UK is restricted by the geographical location of the few centers that are licensed to offer NHS funded PGD. Therefore, PGD is not freely available to all couples at risk. In the Netherlands, exclusion-also known as nondisclosure-PGD is banned by law, so that those who wish to use this approach must travel to a different country such as Belgium. In contrast, the majority of UK PGD uses exclusion.

There is no European registry of prenatal diagnosis for genetic disorders, but the European Society of Human
Reproduction (ESHRE) maintains a PGD registry. The latest report from 2010 found that, of 1574 cycles performed for monogenic disorders, $158(10 \%)$ were for HD. Across the ESHRE data set, the pregnancy rate for PGD was $22 \%$, with 2 of 10 couples undergoing embryo transfer achieving a pregnancy. ${ }^{14}$ HD has become the most common indication for monogenic PGD in the UK, where pregnancy rates are $34 \%$ per cycle, with a live birth rate of $23 \%$ for fresh embryo transfer and $18 \%$ for frozen from 1999 to 2012 (ref. ${ }^{15}$ ). The latest data available from HFEA reported a $25.6 \%$ live birth per initiated cycle for 2013 (ref. ${ }^{16}$ ).

In choosing between PND and PGD couples balance personal, ethical, cultural, and health issues. Many couples consider the concept of PGD as more attractive than PND when planning a future pregnancy because it avoids termination of affected pregnancies and the procedureassociated loss of a normal pregnancy. However, the risks and stresses of PGD also bring their own burdens, coupled with a lower chance of a successful pregnancy outcome ${ }^{17}$ and the extra risks such as hyperstimulation syndrome, surgical egg collection, and obstetric complications. In contrast, the main risk associated with prenatal diagnosis, amniocentesis, or chorionic villus sampling is around a $1 \%$ pregnancy loss risk.

Patterns of CVS and amniocentesis usage are changing rapidly with the advent of noninvasive prenatal screening (NIPS) for screening Down syndrome and other aneuploidies and noninvasive prenatal diagnosis (NIPD) for some monogenic disorders using fetal free DNA. A case of NIPD for HD was recently reported. ${ }^{18}$ Although van den Oever ${ }^{18}$ demonstrated the proof of principle that free fetal DNA can be used for the diagnosis of $\mathrm{HD}$, the intrinsic technical challenges of sequencing for a triplet repeat disorder means that this 
technique is not available in the UK and the majority of European countries. A linkage-based approach may be preferred, as is often used in PGD for $\mathrm{HD}$ and other monogenic disorders. Funding for NIPD in the UK is currently limited.

Further studies of the social and health economic consequences of PND and PGD are required to understand the full effect of these reproductive technologies for the burden of disease in families affected by HD. Our data suggest that a long-term policy of making reproductive technologies available on a population basis free at the point of care has led to a small reduction in HD births in the UK, but the principal motivation for service provision has to remain the wish to support patients and families in facing and coping with this disease. Families with HD require support to face the challenges of this disease and to lead lives, as individuals and families, that are as full and rewarding as can be achieved.

The cost of PND is around $£ 210$ plus clinic costs, and the cost of PGD is around $£ 12,000$ (compared with an IVF cycle cost of around $£ 7500$ ). These costs are easily outweighed by the lifetime medical and social care costs of HD that are averted by decisions to avoid having an affected child. The UK health-care funding model offers couples the opportunity to choose between PND and PGD, and thus we propose that our results reflect couples' wishes for testing when largely unencumbered by financial considerations.

Although testing rates have been captured reliably through the course of the study, detailed data for $12 \%$ of cases are missing due to the challenges of data collection in a nationwide study over three decades and without specific funding. Only minimal and anonymous data were collected, to maintain participation, but PND rates may be slightly underreported. PGD data were obtained directly from all UK centers currently offering PGD. The number of PGD cycles are not equivalent to PND rates because they do not always result in a clinical pregnancy or live birth. The likely level of underascertainment does not alter our conclusions.

In conclusion, this is the longest-running study reporting national rates of prenatal and preimplantation diagnosis for a neurogenetic disorder. Prenatal diagnosis, by both direct and exclusion test methods, is as popular in the UK now as a generation ago. The rate of preimplantation genetic diagnosis for $\mathrm{HD}$ is rising, with many couples seeking exclusion testing. Thus, compared with 20 years ago, more at-risk couples seek to avoid giving birth to children who will later develop HD. Couples including one partner at risk of HD should be offered nondirective information about their reproductive options, prepregnancy advice, and access to both direct and exclusion PND and PGD.

\section{SUPPLEMENTARY INFORMATION}

The online version of this article (https://doi.org/10.1038/s41436018-0367-z) contains supplementary material, which is available to authorized users.

\section{ACKNOWLEDGEMENTS}

We are grateful to UK genetics centers for supplying the data, and Kirsty Mathieson, Vivien Vaughan, and Stella Sihlabela for collating data.

\section{DISCLOSURE}

The authors declare no conflicts of interest.

\section{REFERENCES}

1. Ross CA, Aylward EH, Wild EJ, Langbehn DR, Long JD, Warner JH, et al. Huntington disease: natural history, biomarkers and prospects for therapeutics. Nat Rev Neurol. 2014;10:204-216.

2. Forrest Keenan K, Miedzybrodzka Z, van Teijlingen E, McKee L, Simpson SA. Young people's experiences of growing up in a family affected by Huntington's disease. Clin Genet. 2007;71:120-129.

3. de Die-Smulders CE, de Wert GM, Liebaers I, Tibben A, Evers-Kiebooms G. Reproductive options for prospective parents in families with Huntington's disease: clinical, psychological and ethical reflections. Hum Reprod Update. 2013;19:304-315.

4. The Huntington's Disease Collaborative Research Group. A novel gene containing a trinucleotide repeat that is expanded and unstable on Huntington's disease chromosomes. Cell. 1993;72:971-983.

5. Brezina PR, Kutteh WH. Clinical applications of preimplantation genetic testing. BMJ. 2015;350:g7611

6. Schulman JD, Black SH, Handyside A, Nance WE. Preimplantation genetic testing for Huntington disease and certain other dominantly inherited disorders. Clin Genet. 1996;49:57-58.

7. Simpson SA, Harper PS, United Kingdom Huntington's Disease Prediction Consortium. Prenatal testing for Huntington's disease: experience within the UK 1994-1998. J Med Genet. 2001;38:333-335.

8. Evans SJ, Douglas I, Rawlins MD, Wexler NS, Tabrizi SJ, Smeeth L. Prevalence of adult Huntington's disease in the UK based on diagnoses recorded in general practice records. J Neurol Neurosurg Psychiatry. 2013;84:1156-1160.

9. Simpson SA, Zoeteweij MW, Nys K, Harper P, Dürr A, Jacopini G, et al. Prenatal testing for Huntington's disease: a European collaborative study. Eur J Hum Genet. 2002;10:689-693.

10. Creighton S, Almqvist EW, MacGregor D, Fernandez B, Hogg H, Beis J, et al. Predictive, pre-natal and diagnostic genetic testing for Huntington's disease: the experience in Canada from 1987 to 2000. Clin Genet. 2003:63:462-475.

11. Tassicker RJ, Marshall PK, Liebeck TA, Keville MA, Singaram BM, Richards $\mathrm{FH}$. Predictive and pre-natal testing for Huntington disease in Australia: results and challenges encountered during a 10-year period (1994-2003). Clin Genet. 2006:70:480-489.

12. van Rij MC, de Koning Gans PA, Aalfs CM, Elting M, Ippel PF, Maat-Kievit JA, et al. Prenatal testing for Huntington's disease in the Netherlands from 1998 to 2008. Clin Genet. 2014;85:78-86.

13. van Rij MC, de Koning Gans PA, van Belzen MJ, Roos RA, Geraedts JP, De Rademaeker $M$, et al. The uptake and outcome of prenatal and pre-implantation genetic diagnosis for Huntington's disease in the Netherlands (1998-2008). Clin Genet. 2014;85:87-95.

14. De Rycke M, Belva F, Goossens V, Moutou C, SenGupta SB, TraegerSynodinos J, et al. Consortium data collection XIII: cycles from January to December 2010 with pregnancy follow-up to October 2011. Hum Reprod. 2015:30:1763-1789.

15. Sharpe A, Avery P, Choudhary M Reproductive outcome following pre-implantation genetic diagnosis (PGD) in the UK. Hum Fertil (Camb). 2017;21:1-8. https://doi.org/10.1080/14647273.2017.1336259. Accessed 7 Dec. 2018.

16. Human Embryology Fertility Authority. Fertility treatment 2014: trends and figures. 2016. http://www.hfea.gov.uk/docs/HFEA_Fertility_ treatment_Trends_and_figures_2014.pdf. Accessed 7 Dec. 2018.

17. Miedzybrodzka Z, Templeton A, Dean J, Haites N, Mollison J, Smith N. Preimplantation diagnosis or chorionic villus biopsy? Women's attitudes and preferences. Hum Reprod. 1993;8:2192-2196.

18. van den Oever JM, Bijlsma EK, Feenstra I, Muntjewerff N, Mathijssen IB, Bakker $E$, et al. Non-invasive prenatal diagnosis of Huntington disease: detection of the paternally inherited expanded CAG repeat in maternal plasma. Prenat Diagn. 2015;35:945-949. 